\title{
Long non-coding RNA TUG1 accelerates abnormal growth of airway smooth muscle cells in asthma by targeting the miR-138-5p/E2F3 axis
}

\author{
HAIYIN ZHOU, CAIXIA LONG, PINGPING LIU, YANYING CHEN, LAN LUO and ZHENGHUI XIAO \\ Intensive Care Unit, Hunan Children's Hospital, Changsha, Hunan 410007, P.R. China
}

Received May 27, 2020; Accepted June 28, 2021

DOI: $10.3892 /$ etm.2021.10663

\begin{abstract}
Asthma is a chronic airway inflammatory disease. The present study aimed to explore the effect of the long non-coding RNA taurine-upregulated gene 1 (TUG1) on the viability and migration of airway smooth muscle cells (ASMCs) in asthma. Rat asthma models were constructed with ovalbumin sensitization and challenge and the level of serum immunoglobulin $\mathrm{E}(\mathrm{IgE})$ and the rates of inspiratory and expiratory resistance were measured. Reverse transcription-quantitative PCR was also performed to determine the expression levels of TUG1. Platelet-derived growth factor-BB (PDGF-BB)-treated ASMCs were then used as a cell model of asthma. The viability and migratory abilities of ASMCs were analysed with the MTT and Transwell assays. Additionally, a dual-luciferase reporter assay was used to confirm the relationship between TUG1 and microRNA (miR)-138-5p and between transcription factor E2F3 and miR-138-5p. The expression of TUG1, level of serum IgE, inspiratory resistance and expiratory resistance were clearly increased in the rat asthma model in comparison with controls. Knockdown of TUG1 the viability and migration of PDGF-BB-induced ASMCs and reduced the inspiratory and expiratory resistances. In addition, TUG1 functioned as a bait of miR-138-5p, and miR-138-5p modulated E2F3 expression. Knockdown of E2F3 hindered the abnormal growth of ASMCs. Moreover, miR-138-5p inhibition or E2F3 overexpression reversed the inhibitory effects of TUG1 knockdown on viability and migration of PDGF-BB-induced ASMCs. The TUG1/miR-138-5p/E2F3 regulatory axis appeared to play a critical role in accelerating the viability and migration of ASMCs and may therefore have a role in asthma.
\end{abstract}

Correspondence to: $\mathrm{Dr}$ Zhenghui Xiao, Intensive Care Unit, Hunan Children's Hospital, 86 Ziyuan Road, Changsha, Hunan 410007, P.R. China

E-mail: xiaozhenghui1680@163.com

Key words: asthma, long non-coding RNA TUG1, microRNA-138-5p, E2F3, airway smooth muscle cells

\section{Introduction}

Asthma, a chronic inflammatory disease that is associated with type- 2 cytokines such as IL-4 and IL5, accelerates airway mucus overproduction, eosinophilia, immunoglobulin E (IgE) synthesis and bronchial hyperresponsiveness (1). Migration of airway smooth muscle cells (ASMCs) increases airway smooth muscle mass, to promote airway remodelling, which is a key characteristic of asthma pathogenesis (2). Asthma can be well controlled by an optimized inhaled drug dose and continuous treatment (3). However, asthma often develops into a lifelong condition with a resulting economic burden and may even lead to early permanent disability or premature death (4). It would therefore be beneficial to explore new approaches in the treatment of asthma.

It has been demonstrated that long noncoding RNAs (lncRNAs) exert functions in various cellular processes such as proliferation and migration by regulating DNA, RNA and protein expression levels (5-9). Long noncoding RNA taurine-upregulated genel (TUG1) takes part in a host of respiratory diseases. Silencing of TUG1 has been indicated to regulate LIM domain kinase $2 \mathrm{~b}$ through histone methyl transferase enhancer of zeste homolog 2 , inhibiting cell growth in small cell lung cancer (6). TUG1 was also indicated to promote lung adenocarcinoma cell viability and suppress cell apoptosis (7). TUG1 facilitates pulmonary vascular remodelling through targeting the microRNA (miR)-374c/forkhead box c1 axis in hypoxic pulmonary hypertension (8). The results of recent research also suggest that TUG1 increases the ASMC growth in asthma by regulating the miR-590-5p-mediated FGF1 (9). Therefore, it is hypothesized that TUG1 may play a role in asthma progression.

As biological molecules, miRNAs take part in the development of respiratory diseases including asthma $(10,11)$. miR-217 is expressed at low levels in asthma, and its upregulation suppresses ASMC proliferation and migration by inhibiting zinc finger E-box homeobox 1 expression (12). miR-142 reduces proliferation and induces apoptosis in the ASMCs of asthmatic rats during airway remodelling (13). miR-138 has been demonstrated to attenuate the proliferation of ASMCs from asthmatic donors (14). In previous studies it was suggested that miR-138 is a target of TUG1. Yan et al (15) demonstrated that TUG1 contributed to the development of colorectal cancer via a reduction in miR-138-5p expression and Zhu et al (16) 
reported that TUG1 promoted cervical cancer cell growth and metastasis by suppressing miR-138 expression. However, the specific regulatory relationship between TUG1 and miR-138-5p in asthma remains unclear.

E2F3, a member of the E2F family, participates in the transcriptional activation of genes that regulate the proliferation of both tumour and primary cells (17). Researchers have suggested that E2F3 is associated with the development of respiratory diseases. Cooper et al (18) indicated that small cell lung cancer shows enhanced expression of nuclear staining for E2F3 and Al Ahmed and Nada (19) reported that E2F3 expression increased in lung cancer patients and that overexpression of E2F3 was positively related to metastatic lung cancer. Silencing of lncRNA PVT1 attenuated the viability and migration of ASMCs in asthma by mediating the miR-203a/E2F3 axis (20). However, to the best of our knowledge, the potential regulatory mechanism of TUG1 on the miR-138-5p/ E2F3 axis in asthma has not previously been evaluated.

In the present study, a rat asthma model and a platelet-derived growth factor-BB (PDGF-BB)-treated ASMC cell model were applied. Whether TUG1 controlled the viability and migration of ASMCs via miR-138-5p and E2F3 in asthma was then investigated.

\section{Materials and methods}

Animals. Sprague Dawley rats (female; 60-80 g; 4-weeks-old) were purchased from the Institute of Zoology, Chinese Academy of Sciences. Rats were fed standard chow and water and maintained under a 12 -h light/dark cycle $\left(25^{\circ} \mathrm{C} ; 50 \%\right.$ humidity). This study was performed with the approval of the Animal Ethics Committee of Hunan Children's Hospital (Changsha, China).

Asthmatic rat model establishment. A total of 20 rats were randomly divided into two groups $(n=10)$ : The asthma group and the control group. In the asthma group, rats were intraperitoneally injected with $1 \mathrm{ml} 10 \%$ ovalbumin antigenic suspension (OAS, containing $100 \mathrm{mg}$ ovalbumin and $100 \mathrm{mg}$ aluminium hydroxide (both MilliporeSigma) at 1, 7 and 15 days. On day 21 , the rats were further stimulated by a 7-day ultrasonic atomizing inhalation of $2 \%$ OAS for 30 min daily. Similar procedures were performed on the rats in the control group, but the OAS was replaced with normal saline.

Airway resistance measurement. Inspiratory and expiratory resistances were detected using a rodent ventilator (model 7025; Ugo Basile SRL). Methacholine (MilliporeSigma) at an initial dose of 0.0625 (dissolved in $0.9 \%$ sodium chloride) was intravenously injected into the rats. To obtain a response curve of lung resistance, the dose was increased two-fold with each injection, up to $1 \mathrm{mg} / \mathrm{kg}$. The injection interval was $5 \mathrm{~min}$. Before the next methacholine injection, $50 \mu 1$ methacholine was administered over 4 sec through the return of resistance curves to the pre-methacholine level. Following methacholine administration, the response was detected directly as the peak elevated above the baseline, with the use of AniRes2005 software (Beijing Bestlab High-Tech Co., Ltd.).
PDGF-BB-induced asthma model in ASMCs. Rats were anesthetized with $50 \mathrm{mg} / \mathrm{kg}$ pentobarbital sodium and euthanized by cervical dislocation. ASMCs were isolated from the airway of rats in the control group as previously described (21). The ASMCs were centrifuged $\left(500 \times \mathrm{g}\right.$ for $\left.15 \mathrm{~min} ; 4^{\circ} \mathrm{C}\right)$ and cultured in Dulbecco's modified Eagle's medium (DMEM; Invitrogen; Thermo Fisher Scientific, Inc.) with $10 \%$ foetal bovine serum (FBS; Invitrogen; Thermo Fisher Scientific, Inc.) at $37^{\circ} \mathrm{C}$ and $5 \% \mathrm{CO}_{2}$. The ASMCs were randomly divided into two groups: The PDGF-BB group (ASMCs were stimulated with $25 \mathrm{ng} / \mathrm{ml}$ PDGF-BB for $24 \mathrm{~h}$ ) and the control group (ASMCs without treatment). The PDGF-BB-induced ASMCs acted as the asthmatic model at the cellular level (22).

Enzyme-linked immunosorbent assay (ELISA). After measuring airway reactivity, serum was obtained by lethal cardiac puncture of anesthetized rats. The level of serum IgE in the rats was measured with ELISA using a rat IgE ELISA kit (cat. no. RAB0799MSDS; Sigma Aldrich) according to the manufacturer's guidelines.

Reverse transcription-quantitative polymerase chain reaction $(R T-q P C R)$. Total RNA was extracted from tissues and cells using the TRIzol ${ }^{\circledR}$ reagent (Invitrogen; Thermo Fisher Scientific, Inc.) and was reverse-transcribed into cDNA using the PrimeScript RT reagent kit (Takara Bio, Inc.). PCR reaction was performed on an ABI 7500HT Fast Real-Time PCR System (Applied Biosystems; Thermo Fisher Scientific, Inc.) under the following conditions: $95^{\circ} \mathrm{C}$ for $5 \mathrm{~min}, 40$ cycles of $72^{\circ} \mathrm{C}$ for $34 \mathrm{sec}$ and $60^{\circ} \mathrm{C}$ for $20 \mathrm{sec}$. Relative expression was calculated using the $2^{-\Delta \Delta \mathrm{Cq}}$ method (23). GAPDH, U6 and $\beta$-actin were used for the normalization of TUG1, miR-138-5p and E2F3, respectively. The primer sequences are shown in Table I.

Cell transfection. The short hairpin (sh)-TUG1 (5'-GTCTGC ATTGAGGATATAG-3'), sh-negative control (NC; 5'-CCT CTAGGTAAGCATAAT TTT-3'), miR-138-5p mimics (5'-AGCUGGUGUUGUGAAUCAGGCCG-3'), miR-NC (5'-UUGUACUACACAAAAGUACUG-3'), pcDNA3.1 TUG1 (pcDNA-TUG1) and sh-E2F3 (5'-CATTGAGGTTTACTTATG T-3') were synthesized by Shanghai GenePharma, Co., Ltd. PDGF-BB-induced ASMCs grown to $85 \%$ confluence were transfected or co-transfected with $50 \mathrm{nM}$ of the above-listed agents using Lipofectamine ${ }^{\circledR} 3000$ (Invitrogen; Thermo Fisher Scientific, Inc.) for $4 \mathrm{~h}$ at $37^{\circ} \mathrm{C}$. The ASMCs in the control group did not receive any transfection. Subsequently, $48 \mathrm{~h}$ after transfection, ASMCs were harvested to perform further experiments.

MTT assay. ASMCs and PDGF-BB-treated ASMCs were seeded into 96 -well plates $\left(2 \times 10^{3}\right.$ cells/well) and cultured with $5 \% \mathrm{CO}_{2}$ at $37^{\circ} \mathrm{C}$ for $72 \mathrm{~h}$. Cell viability was then measured using an MTT cell proliferation assay kit (Sigma-Aldrich; Merck $\mathrm{KGaA}$ ), according to the manufacturer's guidelines. In brief, the stock MTT dye solution $(5 \mathrm{mg} / \mathrm{ml}$; Sigma-Aldrich; Merck $\mathrm{KGaA}$ ) was added to each well after the plates were incubated at $37^{\circ} \mathrm{C}$ with $5 \% \mathrm{CO}_{2}$. Following incubation for $2 \mathrm{~h}$, the supernatant was removed and $100 \mu 1$ dimethyl sulfoxide (Sigma-Aldrich; Merck KGaA) was added to each well. The 
Table I. Primer sequences.

\begin{tabular}{ll}
\hline Name of primer & \multicolumn{1}{c}{ Sequences, 5'-3' } \\
\hline TUG1-F & GGACACAATTCGCCACGACTT \\
TUG1-R & GCGCAGTCCCAGATTCCA \\
GAPDH-F & AGAAGGCTGGGGCTCATTTG \\
GAPDH-R & AGGGGCCATCCACAGTCTTC \\
miR-138-5p-F & GCTTAAGGCACGCGG \\
miR-138-5p-R & GTGCAGGGTCCGAGG \\
U6-F & CGCTTCGGCAGCACATATAC \\
U6-R & AAATATGGAACGCTTCACGA \\
E2F3-F & ACAAACAACCAAGACCACAATG \\
E2F3-R & GGGAGGCAGTAAGTTCACAAAC \\
$\beta-$-actin-F & CATGTACGTTGCTATCCAGGC \\
$\beta$-actin-R & CTCCTTAATGTCACGCACGAT
\end{tabular}

miR, microRNA; F, forward; R, reverse.

viability [optical density (OD450)] was analyzed by a Multiskan Spectrum microplate reader (Thermo Fisher Scientific, Inc.).

Transwell assay. Transwell assay was performed to assess the level of cell migration using Transwell chambers $(8-\mu \mathrm{M}$ pore size; Corning Inc.). The lower chamber was filled with $0.6 \mathrm{ml}$ DMEM containing $1 \%$ FBS. The ASMCs and PDGF-BB-treated ASMCs $\left(1 \times 10^{5}\right)$ were suspended in $0.1 \mathrm{ml}$ serum-free DMEM and placed in the upper chamber. After culture for $24 \mathrm{~h}$, the ASMCs and PDGF-BB-treated ASMCs were fixed with $4 \%$ paraformaldehyde at $37^{\circ} \mathrm{C}$ for $1 \mathrm{~h}$ and stained with $0.5 \%$ crystal violet for $10 \mathrm{~min}$ at $37^{\circ} \mathrm{C}$. Cell migration was assessed by counting the cells under a light microscope (magnification, $\mathrm{x} 400$; Olympus Corporation) in five randomly selected views.

Dual-luciferase reporter assay. The potential binding sites between TUG1 and miR-138-5p or miR-138-5p and E2F3 were predicted by Starbase (version 2.0; http://starbase.sysu. edu.cn) or TargetScan (release 7.2; http://www.targetscan. org/vert_72/), respectively. TUG1 and E2F3 with WT or MUT miR-138-5p-binding sites were generated and subcloned into the psiCHECK-2 vectors (Promega Corporation). PDGF-BB-treated ASMCs grew until $85 \%$ confluence and were co-transfected with the relevant luciferase vectors (80 ng) and miR-NC or miR-138-5p mimics (50 nM) using Lipofectamine ${ }^{\circledR} 3000$ at $37^{\circ} \mathrm{C}$ for $4 \mathrm{~h}$. At $48 \mathrm{~h}$ post-incubation, luciferase activity was determined by the dual-luciferase assay system (Promega Corporation). The activity of firefly luciferase was normalized to that of Renilla luciferase.

Lentivirus production and infection. A shRNA-targeted TUG1 and its corresponding scrambled NC were designed and synthesized by Shanghai GenePharma, Co., Ltd. They were inserted into the pGLVU6/Puro vector (Shanghai GenePharma Co., Ltd.) to construct sh-TUG1 and sh-NC. The vectors containing sh-TUG1 or sh-NC together with pMD2.G and psPAX2 plasmids (Shaanxi YouBio Technology Co., Ltd.) were then co-transfected into $293 \mathrm{~T}$ cells using Lipofectamine 3000. Three days post transfection, cell supernatants containing sh-TUG1 or sh-NC lentiviruses were collected. Sh-TUG1 or sh-NC lentiviruses were extracted and purified from cell supernatants. Rats received a tail intravenous injection of $100 \mu \mathrm{l}\left(5 \times 10^{7} \mathrm{TU} / \mathrm{ml}\right)$ sh-TUG1 ( $\mathrm{n}=5$, sh-TUG1 group) or sh-NC ( $n=5$, sh-NC group) lentivirus every $24 \mathrm{~h}$ for three days. At $30 \mathrm{~min}$ after the last injection of lentiviruses, all rats were treated as described previously to establish the asthmatic rat model. After the asthmatic rat model had been established for $24 \mathrm{~h}$, inspiratory and expiratory resistances were detected.

Statistical analysis. Each assay was performed at least three times. Statistical analysis was performed using GraphPad Prism 7.0 (GraphPad Software, Inc.). Data are presented as the mean \pm standard deviation. The differences between two groups or among multiple groups were assessed by Student's t-test or one-way ANOVA followed by Tukey's post hoc test, respectively. $\mathrm{P}<0.05$ was considered to indicate a statistically significant difference.

\section{Results}

TUG1 expression is increased in the airway tissues of the rat asthma model. To determine the association between TUG1 expression and asthma, a rat asthma model was constructed. The results from ELISA indicated that the level of serum IgE was significantly increased in rats in the asthma group compared with the control group $(\mathrm{P}<0.01$; Fig. $1 \mathrm{~A})$. The inspiratory resistance and expiratory resistance were both higher in the asthma group than in the control group $(\mathrm{P}<0.01$; Fig. 1B). Moreover, RT-qPCR revealed that the TUG1 expression was increased in the Asthma group, compared with the control group $(\mathrm{P}<0.01$; Fig. $1 \mathrm{C})$. The results indicated that sh-TUG1 markedly decrease the inspiratory resistance and expiratory resistance of the rat asthma model, compared with the sh-NC group $(\mathrm{P}<0.01$, Fig. 1D).

TUG1 inhibition attenuates the viability and migration of PDGF-BB-induced ASMCs. To establish the asthmatic model at the cellular level, the ASMCs were extracted from the rats and induced by PDGF-BB. As shown in Fig. 2A, TUG1 expression was significantly enhanced in the PDGF-BB-induced ASMCs, compared with the control group $(\mathrm{P}<0.01)$. The expression of TUG1 was significantly reduced by the transfection of sh-TUG1 in ASMCs $(\mathrm{P}<0.01$, Fig. 2B). Furthermore, sh-TUG1 successfully inhibited TUG1 expression in the PDGF-BB-induced ASMCs ( $\mathrm{P}<0.01$; Fig. 2C). MTT and Transwell assays revealed that the viability and migration of ASMCs were considerably increased in the PDGF-BB group, in contrast with the Control group. Moreover, TUG1 knockdown reduced the viability and migration of PDGF-BB-induced ASMCs, compared with the sh-NC group $(\mathrm{P}<0.01$; Fig. 2D and $\mathrm{E})$.

miR-138-5p is a direct target of TUG1. To investigate the mechanism of action underlying the role of TUG1 in PDGF-BB-induced ASMCs, miR-138-5p was identified as a potential target of TUG1 using Starbase (Fig. 3A). Transfection of miR-138-5p mimics markedly increased the expression of miR-138-5p in ASMCs $(\mathrm{P}<0.01$; Fig. 3B). The relative 

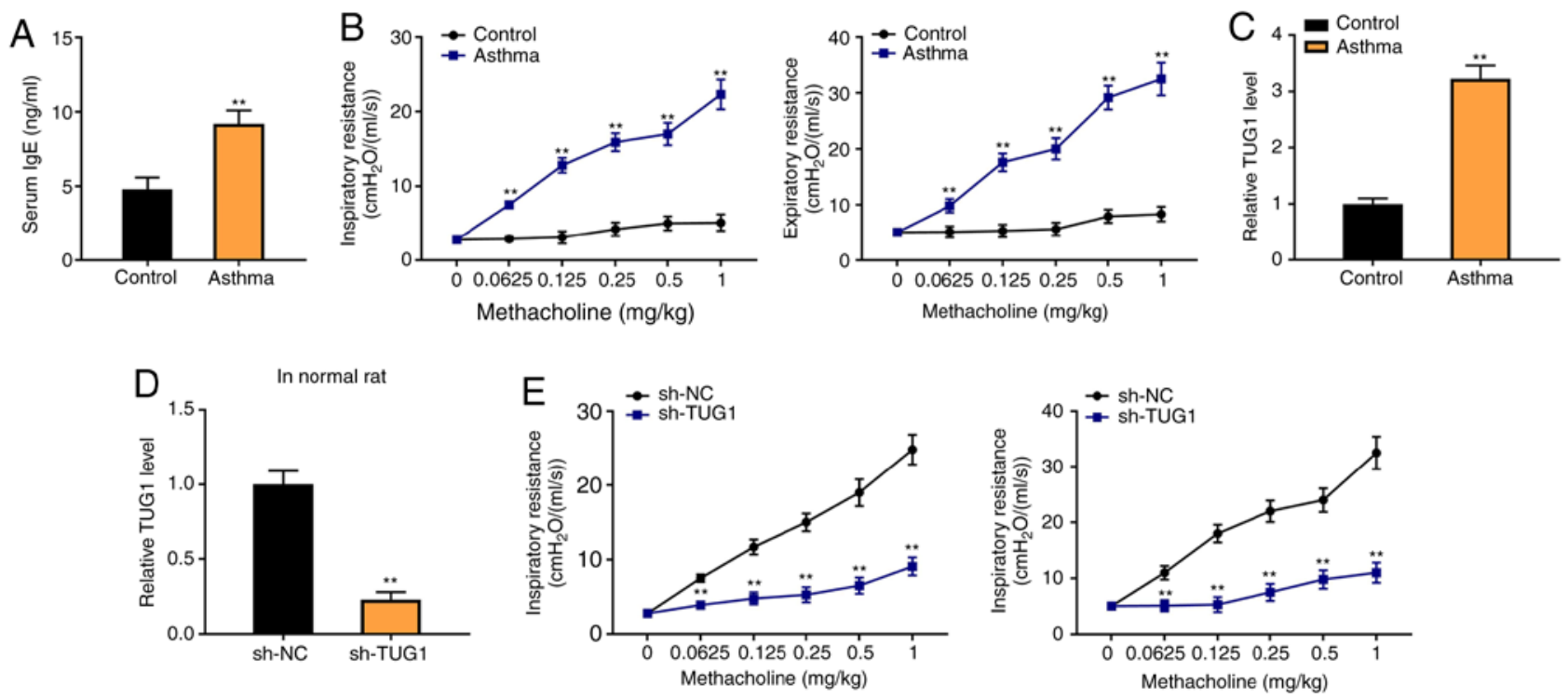

Figure 1. Asthma model establishment is successful and TUG1 expression is increased in the airway tissues of rat asthma model. (A) The level of IgE in the serum isolated from rat asthma model and control was measured by ELISA. (B) The inspiratory resistance and the expiratory resistance of rat asthma model and control. (C) Expression of TUG1 in the rat asthma model and control was detected by RT-qPCR. ${ }^{* *} \mathrm{P}<0.01$ vs. Control. (D) The transfection efficiency of sh-NC and sh-TUG1 was measured by RT-qPCR in the airway tissues of normal rats. ${ }^{* *} \mathrm{P}<0.01$ vs. sh-NC. (E) TUG1 silencing reduced the inspiratory resistance and the expiratory resistance of rat asthma model. ${ }^{* *} \mathrm{P}<0.01$ vs. sh-NC. TUG1, long non-coding RNA taurine-upregulated gene 1 ; sh, short hairpin; NC, negative control; RT-qPCR, reverse transcription-quantitative PCR; IgE, immunoglobulin E.
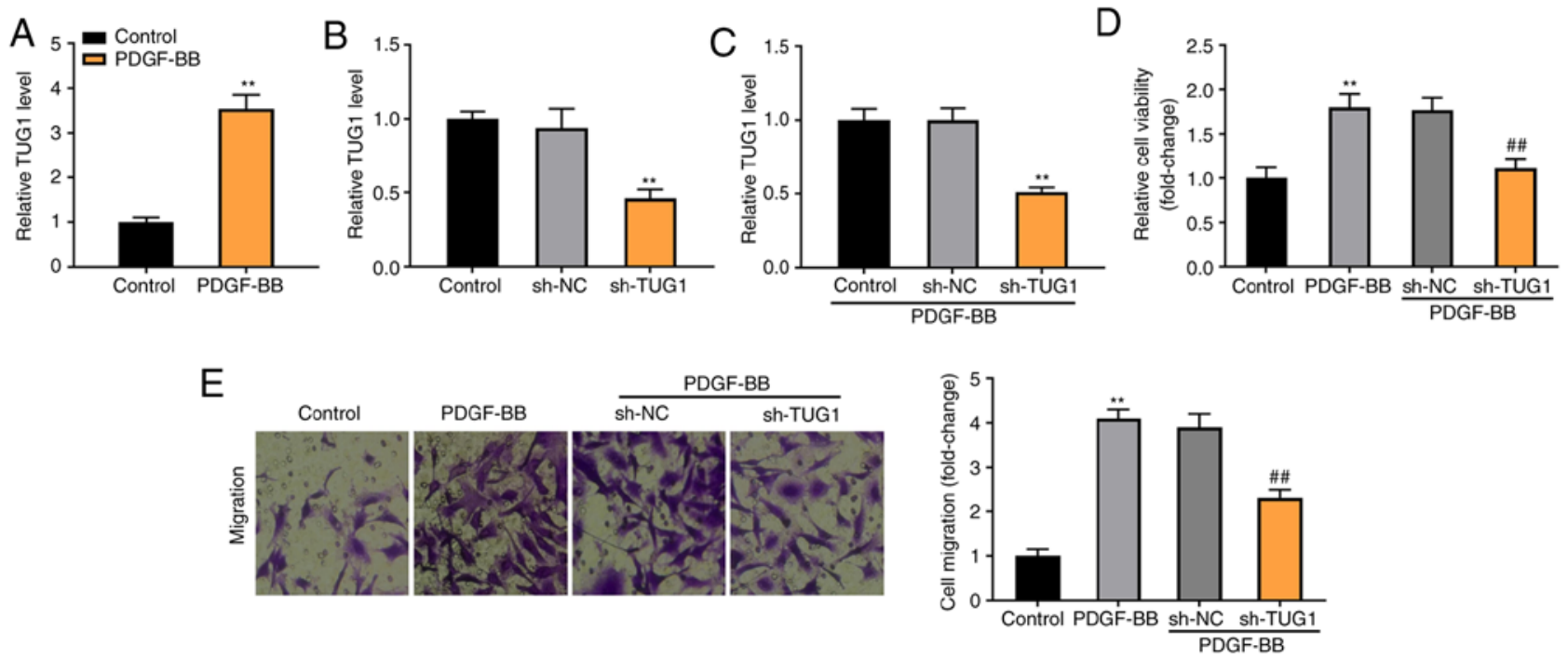

Figure 2. TUG1 inhibition attenuates the viability and migration of PDGF-BB-induced ASMCs. (A) The expression of TUG1 in the PDGF-BB-induced ASMCs and control was detected by reverse transcription-quantitative PCR. ${ }^{* *} \mathrm{P}<0.01$ vs. Control. (B) Reverse transcription-quantitative PCR was performed to measure the transfection efficiency of sh-NC and sh-TUG1 in ASMCs. ${ }^{* *} \mathrm{P}<0.01$ vs. sh-NC. (C) Knockdown of TUG1 was performed using sh-TUG1 transfection. ${ }^{* *} \mathrm{P}<0.01$ vs. sh-NC. (D) The viability of PDGF-BB-induced ASMCs and control was measured by MTT assay. ${ }^{* *} \mathrm{P}<0.01$ vs. Control; ${ }^{\# \#} \mathrm{P}<0.01$ vs. sh-NC. (E) Migration of PDGF-BB-induced ASMCs and control was tested by Transwell assay. Magnification, $\mathrm{x} 400 .{ }^{* *} \mathrm{P}<0.01 \mathrm{vs}$. Control, ${ }^{\# \#} \mathrm{P}<0.01 \mathrm{vs}$. sh-NC. PDGF-BB, platelet-derived growth factor-BB; ASMC, airway smooth muscle cell; TUG1, long non-coding RNA taurine-upregulated gene 1; sh, short hairpin; NC, negative control.

luciferase activity was decreased in ASMCs co-transfected with miR-138-5p mimics and TUG1 Wt, compared with cells co-transfected with miR-NC and TUG1 Wt (P<0.01; Fig. 3C). As shown in Fig. 3D, downregulation of TUG1 markedly increased miR-138-5p expression $(\mathrm{P}<0.01)$.

miR-138-5p decreases the viability and migration of PDGF-BB-induced ASMCs. As shown in Fig. 4A, expression of miR-138-5p was notably downregulated in the PDGF-BB group, compared with the Control group $(\mathrm{P}<0.01)$. To verify the regulatory effect of miR-138-5p on PDGF-BB-induced ASMCs, miR-138-5p expression was increased by the transfection of miR-138-5p mimics into ASMCs (P<0.01, Fig. 4B). As shown in Fig. $4 \mathrm{C}$ and $\mathrm{D}$, the viability and migration of ASMCs were markedly enhanced in the PDGF-BB group compared with the Control group $(\mathrm{P}<0.01)$. The transfection 
A TUG1 wt 5' acugagcaagCACUACCACCAGCa 3' miR-138-5p 3' gccggacuaaGUG-UUGUGGUCGa $5^{\prime}$ TUG1 mut $5^{\prime}$ acugagcaagGUGUUCGUGGUCGa 3'
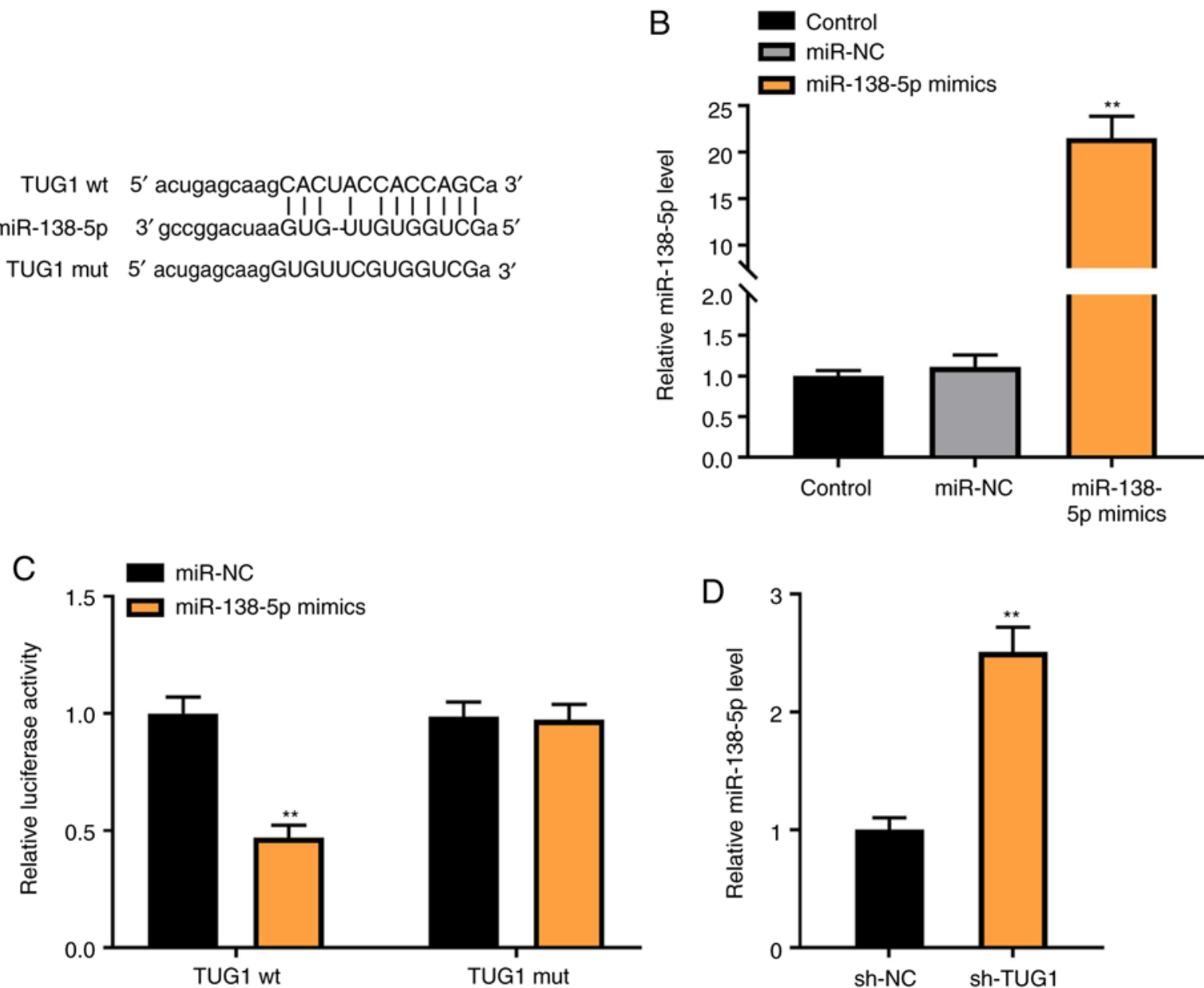

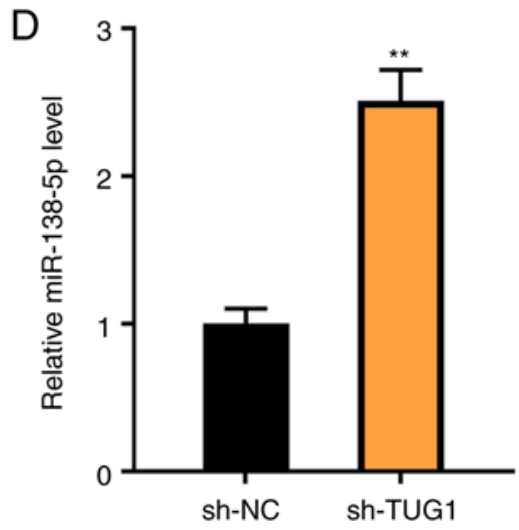

Figure 3. miR-138-5p is a direct target of TUG1. (A) Starbase identified the predicted binding site between TUG1 and miR-138-5p. (B) The transfection efficiency of miR-NC and miR-138-5p were measured by RT-qPCR in ASMCs. ${ }^{* * *} \mathrm{P}<0.01$ vs. sh-NC. (C) Relative luciferase activity in PDGF-BB-induced ASMCs was measured by dual-luciferase reporter assay. ${ }^{* *} \mathrm{P}<0.01$ vs. miR-NC. (D) The expression of miR-138-5p in PDGF-BB-induced ASMCs was detected by RT-qPCR. ${ }^{* *}$ P $<0.01$ vs. sh-NC. PDGF-BB, platelet-derived growth factor-BB; ASMC, airway smooth muscle cell; TUG1, long non-coding RNA taurine-upregulated gene 1; sh, short hairpin; NC, negative control; miR, microRNA; wt, wild type; mut, mutant; RT-qPCR, reverse transcription-quantitative PCR.

of miR-138-5p mimics notably decreased the viability and migration of PDGF-BB-induced ASMCs compared with the miR-NC group $(\mathrm{P}<0.01)$.

miR-138-5p modulates E2F3 expression levels. To demonstrate the underlying mechanism by which TUG1 mediated the growth of ASMCs, TargetScan was used to predict the binding site for miR-138-5p on the 3' UTR of E2F3 (Fig. 5A). Using the luciferase reporter assay, it was determined that miR-138-5p overexpression considerably inhibited the luciferase activity of WT E2F3 3' UTR reporter vector ( $\mathrm{P}<0.01$; Fig. 5B). As shown in Fig. 5C, the E2F3 expression in the PDGF-BB group was higher than that in the control group $(\mathrm{P}<0.01)$. Transfection of miR-138-5p mimics notably decreased the E2F3 expression in PDGF-BB-induced ASMCs, compared with the miR-NC group $(\mathrm{P}<0.01)$.

Knockdown of E2F3 decreases the viability and migration of PDGF-BB-induced ASMCs. To explore the molecular mechanism by which E2F3 regulates the viability and migration of PDGF-BB-induced ASMCs, E2F3 was knocked down by the transfection of sh-E2F3 (P<0.01; Fig. 6A). Knockdown of E2F3 significantly decreased the expression of E2F3 in
PDGF-BB-induced ASMCs ( $\mathrm{P}<0.01$; Fig. 6B). As illustrated in Fig. $6 \mathrm{C}$ and D, the MTT and Transwell assays revealed that the viability and migration of ASMCs were higher in the PDGF-BB group compared with the control group $(\mathrm{P}<0.01)$, and knockdown of E2F3 visibly decreased the viability and migration of PDGF-BB-induced ASMCs compared with the sh-NC group $(\mathrm{P}<0.01)$.

Knockdown of TUG1 decreases the viability and migration of PDGF-BB-induced ASMCs via regulating miR-138-5p/E2F3 axis. $\mathrm{E} 2 \mathrm{~F} 3$ expression was enhanced by the transfection of pcDNA-E2F3 in both ASMCs and FDGF-BB-induced ASMCs $(\mathrm{P}<0.01$; Fig. 7A and B). miR-138-5p expression was inhibited by the transfection of miR-138-5p inhibitor in both ASMCs and FDGF-BB-induced ASMCs ( $\mathrm{P}<0.01$; Fig. $7 \mathrm{C}$ and $\mathrm{D})$. To verify whether TUG1 regulated the miR-138-5p/E2F3 axis in asthma, a feedback experiment was performed using MTT and Transwell assay in PDGF-BB-induced ASMCs. As illustrated in Fig. 7C and $\mathrm{D}$, TUG1 knockdown visibly reduced the viability and migration of PDGF-BB-induced ASMCs $(\mathrm{P}<0.01)$. Moreover, miR-138-5p inhibition or E2F3 overexpression partially reversed the inhibitory effects of sh-TUG1 on the viability and migration of PDGF-BB-induced ASMCs $(\mathrm{P}<0.01)$. 
A

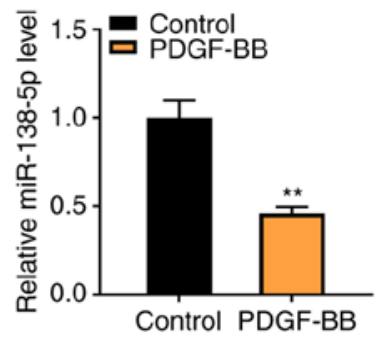

B

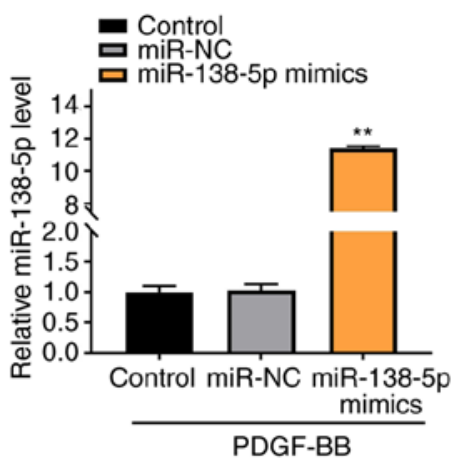

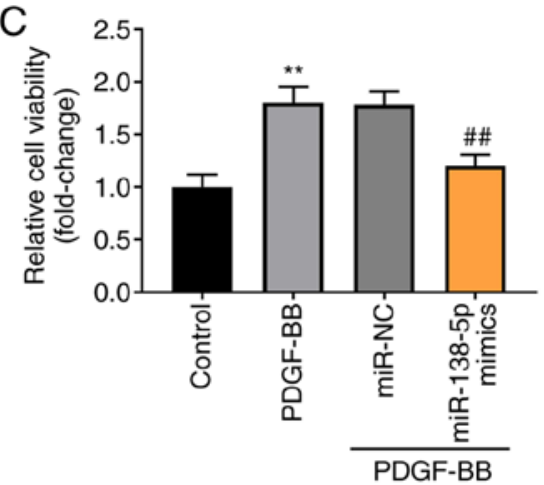

D

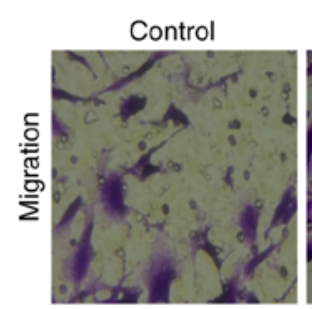

PDGF-BB

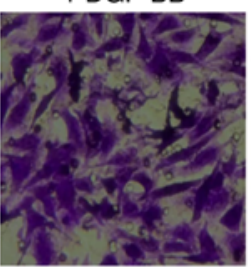

PDGF-BB

miR-NC

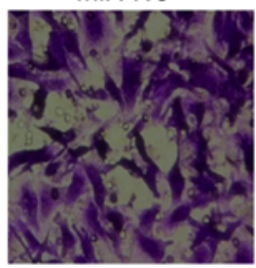

miR-138-5

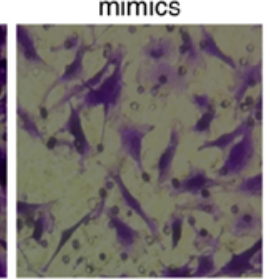

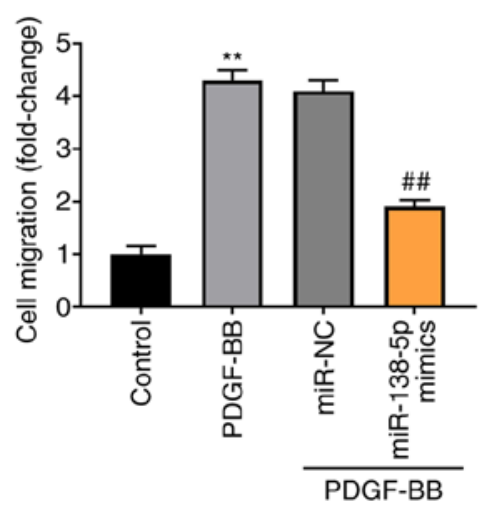

Figure 4. miR-138-5p decreases the viability and migration of PDGF-BB-induced ASMCs. (A) The expression of miR-138-5p in the PDGF-BB-induced ASMCs and control was detected by reverse transcription-quantitative PCR. ${ }^{* *} \mathrm{P}<0.01$ vs. Control. (B) Overexpression of miR-138-5p was constructed via miR-138-5p mimics transfection. ${ }^{* *} \mathrm{P}<0.01$ vs. miR-NC. (C) The viability of PDGF-BB-induced ASMCs and control was measured via MTT assay. ${ }^{* *} \mathrm{P}<0.01$ vs. Control; ${ }^{\# \#} \mathrm{P}<0.01$ vs. miR-NC. (D) The migration of PDGF-BB-induced ASMCs and control was tested by Transwell assay. Magnification, $\mathrm{x} 400 .{ }^{* *} \mathrm{P}<0.01 \mathrm{vs}$. Control; ${ }^{\# \prime} \mathrm{P}<0.01$ vs. miR-NC. PDGF-BB, platelet-derived growth factor-BB; ASMC, airway smooth muscle cell; TUG1, long non-coding RNA taurine-upregulated gene 1; NC, negative control; miR, microRNA.

\section{A}

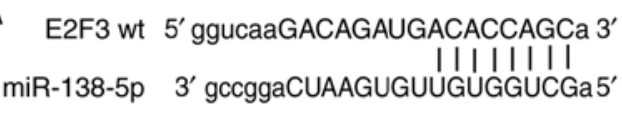
E2F3 mut $5^{\prime}$ ggucaaGACAGAUGUGUGGUCGa $3^{\prime}$
B

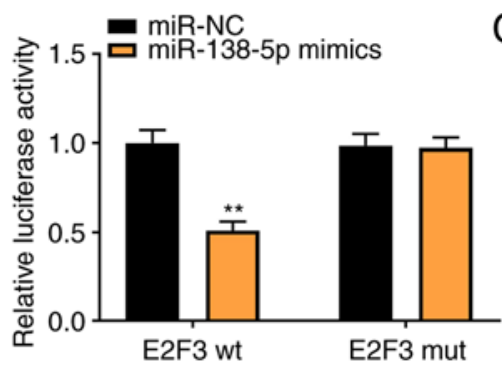

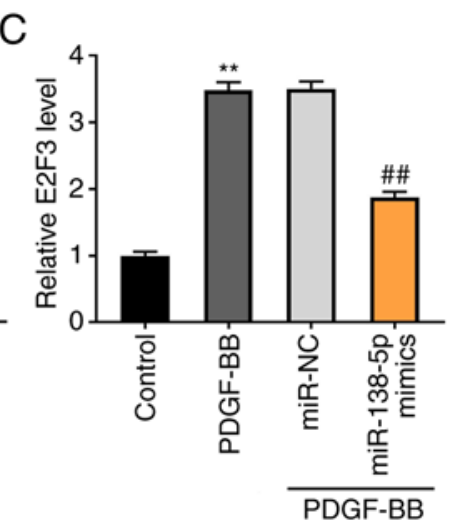

Figure 5. miR-138-5p modulates E2F3 expression. (A) TargetScan showed the predicted binding site between E2F3 and miR-138-5p. (B) Relative luciferase activity in PDGF-BB-induced ASMCs was measured via dual-luciferase reporter assay. ${ }^{* *} \mathrm{P}<0.01$ vs. miR-NC. (C) The expression of E2F3 in PDGF-BB-induced ASMCs and control was detected by reverse transcription-quantitative PCR. ${ }^{* *} \mathrm{P}<0.01$ vs. Control, ${ }^{* \#} \mathrm{P}<0.01$ vs. miR-NC. PDGF-BB, platelet-derived growth factor-BB; ASMC, airway smooth muscle cell; TUG1, long non-coding RNA taurine-upregulated gene 1; NC, negative control; miR, microRNA; wt, wild type; mut, mutant.

\section{Discussion}

It has been documented that airway remodelling accelerates structural changes to the airway in patients with asthma, leading to irreversible or partially irreversible airflow obstruction (24).
In the present study, the level of serum IgE, inspiratory resistance and expiratory resistance were increased in a rat asthma model. Previous studies have confirmed that the serum $\operatorname{lgE}$ level and airway resistance are both elevated in asthma model rats $(25,26)$. These data indicated that the rat asthma model 

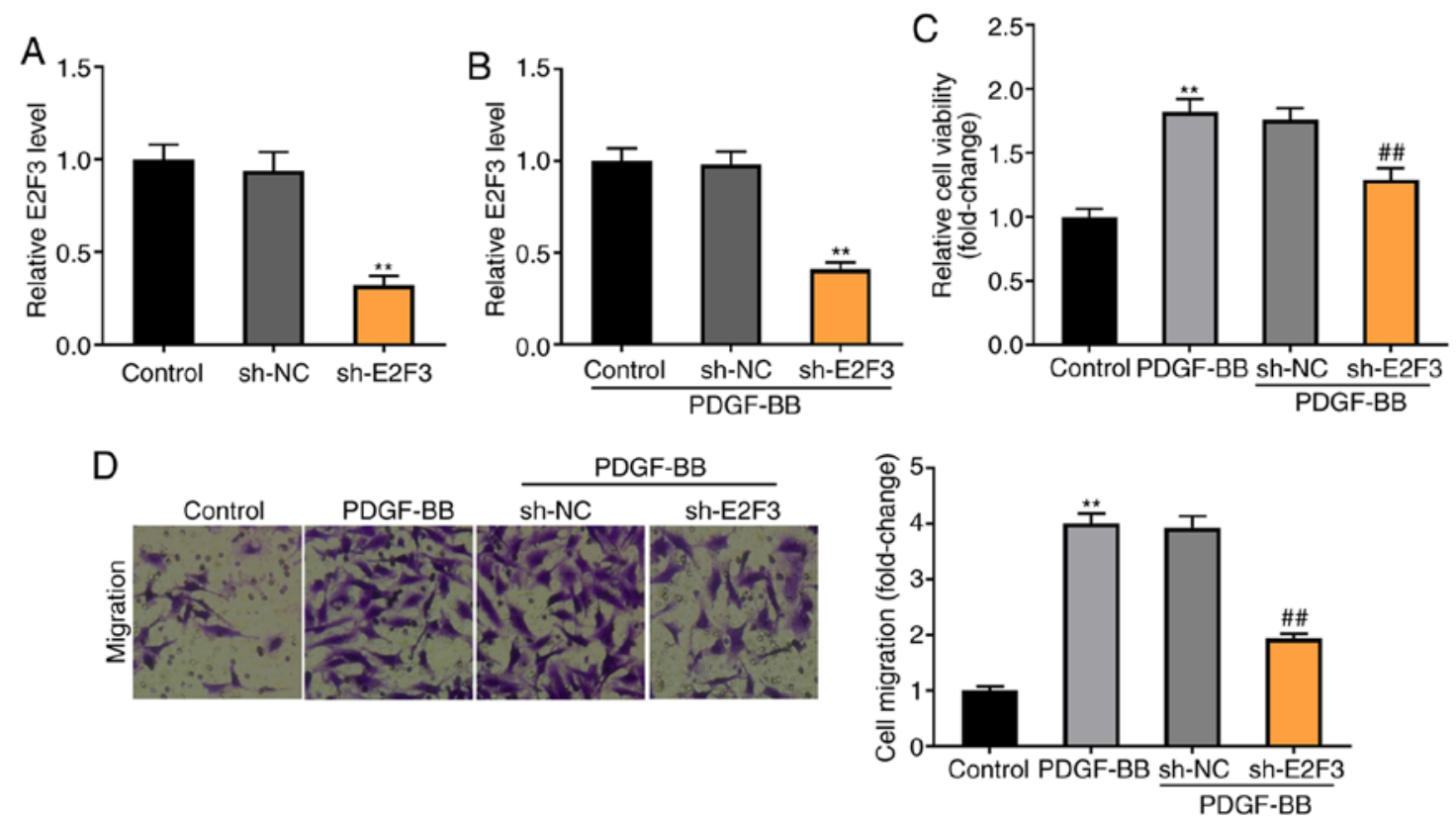

Figure 6. Knockdown of E2F3 decreases the viability and migration of PDGF-BB-induced ASMCs. (A) Reverse transcription-quantitative PCR was performed to detect the transfection efficiency of sh-NC and sh-E2F3 in ASMCs. ${ }^{* *} \mathrm{P}<0.01$ vs. sh-NC. (B) Knockdown of E2F3 was performed via sh-E2F3 transfection. ${ }^{* *} \mathrm{P}<0.01$ vs. sh-NC. (C) The viability of PDGF-BB-induced ASMCs and control was measured by MTT assay. ${ }^{* *} \mathrm{P}<0.01$ vs. Control; ${ }^{\# \#} \mathrm{P}<0.01 \mathrm{vs}$. sh-NC. (D) The migration of PDGF-BB-induced ASMCs and control was examined via Transwell assay. Magnification, $\mathrm{x} 400 .{ }^{* *} \mathrm{P}<0.01$ vs. Control, ${ }^{\# \#} \mathrm{P}<0.01 \mathrm{vs}$. sh-NC. PDGF-BB, platelet-derived growth factor-BB; ASMC, airway smooth muscle cell; TUG1, long non-coding RNA taurine-upregulated gene 1; NC, negative control; miR, microRNA; sh, short hairpin.
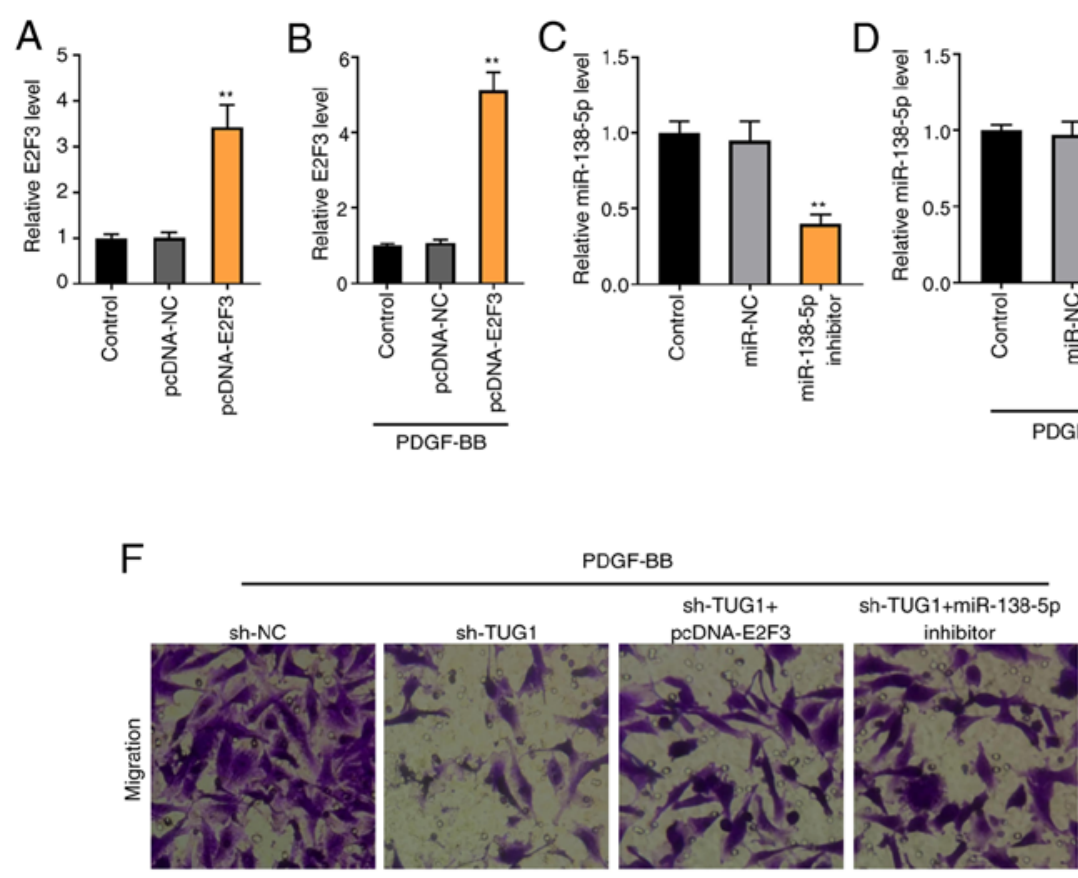
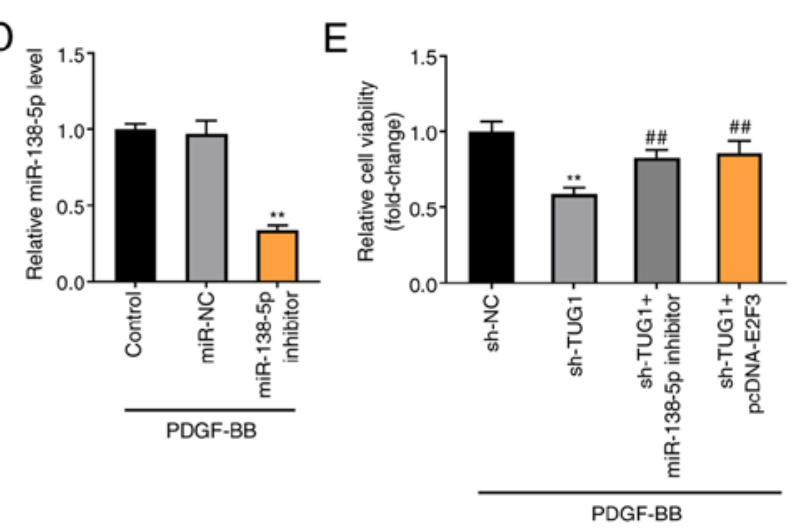

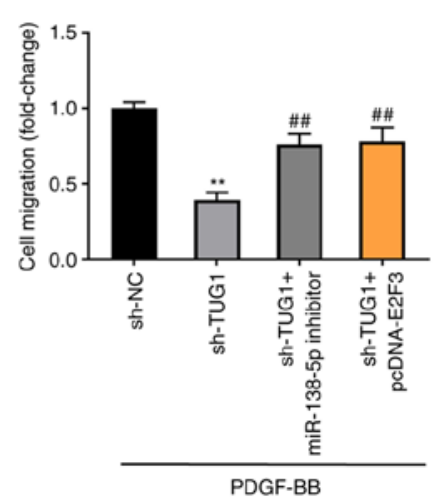

Figure 7. Knockdown of TUG1 decreased the viability and migration of PDGF-BB-induced ASMCs via regulating the miR-138-5p/E2F3 axis. (A) The transfection efficiency of pcDNA-NC and pcDNA-E2F3 were measured by RT-qPCR in ASMCs. ${ }^{* *} \mathrm{P}<0.01$ vs. pcDNA-NC. (B) Overexpression of E2F3 was constructed via pcDNA-E2F3 transfection. ${ }^{* *} \mathrm{P}<0.01 \mathrm{vs}$. pcDNA-NC. (C) RT-qPCR was performed to detect the transfection efficiency of miR-NC and miR-138-5p inhibitor in ASMCs. ${ }^{* *} \mathrm{P}<0.01$ vs. miR-NC. (D) Inhibition of miR-138-5p was established using miR-138-5p inhibitor transfection. ${ }^{* *} \mathrm{P}<0.01$ vs. miR-NC. (E) The viability of PDGF-BB-induced ASMCs was measured by MTT assay. ${ }^{* *} \mathrm{P}<0.01$ vs. sh-NC; ${ }^{\# \#} \mathrm{P}<0.01$ vs. sh-TUG1. (F) The migration of PDGF-BB-induced ASMCs was tested by Transwell assay. magnification, $\mathrm{x} 400 .{ }^{* *} \mathrm{P}<0.01$ vs. sh-NC; ${ }^{\# \#} \mathrm{P}<0.01$ vs. sh-TUG1. PDGF-BB, platelet-derived growth factor-BB; ASMC, airway smooth muscle cell; TUG1, long non-coding RNA taurine-upregulated gene 1; NC, negative control; miR, microRNA; sh, short hairpin. 
was constructed successfully in the present study. Enhanced expression levels of lncRNAs, including lncRNA TCF7 (27), PVT1 (28) and lncRNA LINC00882 (29), have been discovered in cases of asthma. In the present study, TUG1 expression was elevated in both the rat asthma model and in PDGF-BB-induced ASMCs. Taking these results into consideration, it can be hypothesized that TUG1 may be a regulator of asthma.

Results from previous studies suggest that abnormal growth of ASMCs leads to airway re-modelling in asthma via thickening of the airway wall $(30,31)$. IncRNAs may serve as critical factors in the acceleration of the asthma process. Zhang et al (26) suggested that 1ncRNA GAS5 binds to miR-10a to upregulate BDNF expression, promoting ASMC proliferation in asthma. Fan et al (27) demonstrated that 1ncRNA TCF7 interacts with TIMMDC1 to facilitate ASMC growth and migration in asthma by mediating AKT. Lin et al (9) reported that TUG1 contributed to the proliferation and migration of ASMCs in asthma through modulation of the miR-590-5p/FGF1 axis. In the present study, TUG1 knockdown reduced the viability and migration of PDGF-BB-induced ASMCs. The role of TUG1 was similar to that of the aforementioned lncRNAs and suggests that TUG1 may be a potential therapeutic target for asthma. To verify the anti-asthma effect of TUG1 inhibition in vivo, an asthmatic rat model was established. It was demonstrated that TUG1 knockdown inhibited inspiratory and expiratory resistance in the model rats. These data illustrate that TUG1 inhibition may alleviate the progression of asthma in a rat model.

Several lncRNAs interact with miRNAs to regulate the progression of asthma. Downregulation of lncRNA BCYRN1 interacts with miR-150 to promote abnormal growth of ASMCs in asthmatic rats (32). Silencing of lncRNA Malat1 suppresses proliferation and migration of PDGF-BB-induced ASMCs by endogenously competing against miR-150 (33). In the present study, miR-138-5p was demonstrated to be a target of TUG1. An increasing number of studies have shown that miR-138 is downregulated and serves a key role in the regulation of asthma. miR-138 mediates a balance of Th1/Th2 and attenuates $\mathrm{CD}^{+}{ }^{+} \mathrm{T}$ cell proliferation by targeting $\mathrm{OX} 40 \mathrm{~L}$, which may inhibit the progression of asthma (34). miR-138 expression is decreased in human ASMCs, and miR-138 upregulation inhibits the proliferation of ASMCs (14). In the present study decreased miR-138-5p expression was observed in PDGF-BB-induced ASMCs, and miR-138-5p inhibition partially reversed the inhibitory effects of TUG1 knockdown on viability and migration of PDGF-BB-induced ASMCs. These results demonstrated that TUG1 can promote the abnormal growth of ASMCs via inhibition miR-138-5p in asthma.

It has been documented that the expression of genes in the E2F family is increased and these genes regulate the progression of respiratory diseases. Inhibition of E2F-1 gene expression can attenuate ASMC proliferation in asthma (35). E2F3 is highly expressed, and knockdown of E2F3 reduces cell growth arrest and senescence in lung cancer (36). In the present study, loss-of-function experiments revealed that silencing of E2F3 reduced viability and migration of PDGF-BB-treated ASMCs, suggesting that E2F3 silencing may attenuate the abnormal growth of ASMCs. Prior studies have reported that lncRNAs serve as competing endogenous
RNAs to inhibit miRNA-mediated target gene downregulation in the pathogenesis of asthma $(37,38)$. IncRNA PVT1 interacts with miR-203a to promote ASMC proliferation and migration by increasing E2F3 (20). TUG1 binds to miR-590-5p to increase FGF1 expression, facilitating abnormal ASMC growth in asthma (9). In the current study, E2F3 was targeted by miR-138-5p, and miR-138-5p overexpression decreased E2F3 expression in PDGF-BB-stimulated ASMCs. Considering the interaction between TUG1 and miR-138-5p, it can be hypothesized that TUG1 knockdown may inhibit E2F3 expression by increasing miR-138-5p expression in PDGF-BB-stimulated ASMCs. Moreover, feedback experiments indicated that E2F3 overexpression reversed the inhibitory effects of TUG1 knockdown on viability and migration of PDGF-BB-induced ASMCs. Taken together, these data suggest that TUG1 may promote the viability and migration of ASMCs in asthma by regulating the miR-138-5p/E2F3 axis.

In conclusion, TUG1 expression was upregulated in asthmatic rats, and knockdown of TUG1 attenuated the progression of asthma. Furthermore, TUG1 may contribute to the abnormal growth of ASMCs by regulating the miR-138-5p/E2F3 axis. Therefore, TUG1 may represent a promising therapeutic target for asthma.

\section{Acknowledgements}

Not applicable.

\section{Funding}

The present study was supported by grants from the National Science and Technology Support Plan for the 12th Five-Year Plan Period (grant no. 2012BAI04B01), the Hunan Key Laboratory of Pediatric Emergency Medicine (grant no. 2018TP1028) and the Hunan Provincial Health Commission Project (grant no. 20200611).

\section{Availability of data and materials}

The datasets used and/or analyzed during the current study are available from the corresponding author on reasonable request.

\section{Authors' contributions}

HZ conceived and designed the study, performed experiments and analyzed the data and drafted the manuscript. CL performed experiments and drafted the manuscript. PL, YC, LL and ZX performed experiments and critically revised the manuscript. HZ and CL confirm the authenticity of all the raw data. All authors read and approved the final manuscript for publication.

\section{Ethics approval and consent to participate}

Ethical approval was obtained from the Animal Ethics Committee of Hunan Children's Hospital.

\section{Patient consent for publication}

Not applicable. 


\section{Competing interests}

The authors declare that they have no competing interests.

\section{References}

1. Lambrecht BN, Hammad H and Fahy JV: The cytokines of asthma. Immunity 50: 975-991, 2019.

2. Salter B, Pray C, Radford K, Martin JG and Nair P: Regulation of human airway smooth muscle cell migration and relevance to asthma. Respir Res 18: 156, 2017.

3. Israel E and Reddel HK: Severe and difficult-to-treat asthma in adults. N Engl J Med 377: 965-976, 2017.

4. Nunes C, Pereira AM and Morais-Almeida M: Asthma costs and social impact. Asthma Res Pract 3: 1, 2017.

5. Turner M, Galloway A and Vigorito E: Noncoding RNA and its associated proteins as regulatory elements of the immune system. Nat Immunol 15: 484-491, 2014.

6. Niu Y, Ma F, Huang W, Fang S, Li M, Wei T and Guo L: Long non-coding RNA TUG1 is involved in cell growth and chemoresistance of small cell lung cancer by regulating LIMK2b via EZH2. Mol Cancer 16: 5, 2017.

7. Liu H, Zhou G, Fu X, Cui H, Pu G, Xiao Y, Sun W, Dong X, Zhang L, Cao S, et al: Long noncoding RNA TUG1 is a diagnostic factor in lung adenocarcinoma and suppresses apoptosis via epigenetic silencing of BAX. Oncotarget 8: 101899-101910, 2017.

8. Yang L, Liang H, Shen L, Guan Z and Meng X: LncRNA Tug1 involves in the pulmonary vascular remodeling in mice with hypoxic pulmonary hypertension via the microRNA374c-mediated Foxc1. Life Sci 237: 116769, 2019.

9. Lin J, Feng X, Zhang J and Tong Z: Long noncoding RNA TUG1 promotes airway smooth muscle cells proliferation and migration via sponging miR-590-5p/FGF1 in asthma. Am J Transl Res 11: 3159-3166, 2019.

10. Yoo JK, Lee JM, Kang SH, Jeon SH, Kim CM, Oh SH, Kim CH, Kim NK and Kim JK: The novel microRNA hsa-miR-CHA1 regulates cell proliferation and apoptosis in human lung cancer by targeting XIAP. Lung Cancer 132: 99-106, 2019.

11. Zhang Y, Xue Y, Liu Y, Song G, Lv G, Wang Y, Wang Y, Li X and Yang L: MicroRNA-146a expression inhibits the proliferation and promotes the apoptosis of bronchial smooth muscle cells in asthma by directly targeting the epidermal growth factor receptor. Exp Ther Med 12: 854-858, 2016.

12. Gao Y, Wang B, Luo H, Zhang Q and Xu M: miR-217 represses TGF- $\beta 1$-induced airway smooth muscle cell proliferation and migration through targeting ZEB1. Biomed Pharmacother 108: 27-35, 2018.

13. Wang J, Wang HS and Su ZB: MicroRNA-142 inhibits proliferation and promotes apoptosis in airway smooth muscle cells during airway remodeling in asthmatic rats via the inhibition of TGF- $\beta$-dependent EGFR signaling pathway. Cell Physiol Biochem 47: 1682-1695, 2018.

14. Liu Y, Yang K, Sun X, Fang P, Shi H, Xu J, Xie M and Li M: MiR-138 suppresses airway smooth muscle cell proliferation through the PI3K/AKT signaling pathway by targeting PDK1. Exp Lung Res 41: 363-369, 2015.

15. Yan Z, Bi M, Zhang Q, Song Y and Hong S: LncRNA TUG1 promotes the progression of colorectal cancer via the miR-138-5p/ZEB2 axis. Biosci Rep 40: BSR20201025, 2020.

16. Zhu J, Shi H, Liu H, Wang X and Li F: Long non-coding RNA TUG1 promotes cervical cancer progression by regulating the miR-138-5p-SIRT1 axis. Oncotarget 8: 65253-65264, 2017.

17. Humbert PO, Verona R, Trimarchi JM, Rogers C, Dandapani S and Lees JA: E2F3 is crucial for normal cellular proliferation. Genes Dev 14: 690-703, 2000.

18. Cooper CS, Nicholson AG, Foster C, Dodson A, Edwards S, Fletcher A, Roe T, Clark J, Joshi A, Norman A, et al: Nuclear overexpression of the E2F3 transcription factor in human lung cancer. Lung Cancer 54: 155-162, 2006.

19. Al Ahmed HA and Nada O: E2F3 transcription factor: A promising biomarker in lung cancer. Cancer Biomark 19: 21-26, 2017.

20. Yu X, Zhe Z, Tang B, Li S, Tang L, Wu Y, Chen X and Fang H: $\alpha$-Asarone suppresses the proliferation and migration of ASMCs through targeting the lncRNA-PVT1/miR-203a/E2F3 signal pathway in RSV-infected rats. Acta Biochim Biophys Sin (Shanghai) 49: 598-608, 2017.
21. Chen $\mathrm{G}$ and Khalil N: TGF-beta1 increases proliferation of airway smooth muscle cells by phosphorylation of map kinases. Respir Res 7: 2, 2006.

22. Zhou H, Wu Q, Wei L and Peng S: Paeoniflorin inhibits PDGF-BB-induced human airway smooth muscle cell growth and migration. Mol Med Rep 17: 2660-2664, 2017.

23. Livak KJ and Schmittgen TD: Analysis of relative gene expression data using real-time quantitative PCR and the 2(-Delta Delta C(T) method. Methods 25: 402-408, 2001.

24. Najafi A, Masoudi-Nejad A, Ghanei M, Nourani MR and Moeini A: Pathway reconstruction of airway remodeling in chronic lung diseases: A systems biology approach. PLoS One 9: e100094, 2014

25. Zhang XY, Zhang LX, Tian CJ, Tang XY, Zhao LM, Guo YL, Cheng DJ, Chen XL, Ma LJ and Chen ZC: LncRNAs $\mathrm{BCYRN1}$ promoted the proliferation and migration of rat airway smooth muscle cells in asthma via upregulating the expression of transient receptor potential 1. Am J Transl Res 8: 3409-3418, 2016.

26. Zhang XY, Tang XY, Li N, Zhao LM, Guo YL, Li XS, Tian CJ, Cheng DJ, Chen ZC and Zhang LX: GAS5 promotes airway smooth muscle cell proliferation in asthma via controlling miR-10a/BDNF signaling pathway. Life Sci 212: 93-101, 2018.

27. Fan M, Xu J, Xiao Q, Chen F and Han X: Long non-coding RNA TCF7 contributes to the growth and migration of airway smooth muscle cells in asthma through targeting TIMMDC1/Akt axis. Biochem Biophys Res Commun 508: 749-755, 2019.

28. Austin PJ, Tsitsiou E, Boardman C, Jones SW, Lindsay MA, Adcock IM, Chung KF and Perry MM: Transcriptional profiling identifies the long noncoding RNA plasmacytoma variant translocation (PVT1) as a novel regulator of the asthmatic phenotype in human airway smooth muscle. J Allergy Clin Immunol 139: 780-789, 2017.

29. Liu Z, Mei L and He Z: Long non-coding RNA00882 contributes to platelet-derived growth factor-induced proliferation of human fetal airway smooth muscle cells by enhancing Wnt/beta-catenin signaling via sponging miR-3619-5p. Biochem Biophys Res Commun 514: 9-15, 2019

30. Keglowich LF and Borger P: The three A's in asthma-airway smooth muscle, airway remodeling \& angiogenesis. Open Respir Med J 9: 70-80, 2015.

31. Perry MM, Baker JE, Gibeon DS, Adcock IM and Chung KF: Airway smooth muscle hyperproliferation is regulated by microRNA-221 in severe asthma. Am J Respir Cell Mol Biol 50: 7-17, 2014.

32. Zhang XY, Tang XY, Ma LJ, Guo YL, Li XS, Zhao LM, Tian CJ, Cheng DJ, Chen ZC and Zhang LX: Schisandrin B down-regulated lncRNA BCYRN1 expression of airway smooth muscle cells by improving miR- 150 expression to inhibit the proliferation and migration of ASMC in asthmatic rats. Cell Prolif 50: e12382, 2017.

33. Lin L, Li Q, Hao W, Zhang Y, Zhao L and Han W: Upregulation of LncRNA Malat1 induced proliferation and migration of airway smooth muscle cells via miR-150-eIF4E/Akt signaling. Front Physiol 10: 1337, 2019.

34. Huang L, Wang M, Chen Z, Yan Y, Gu W, Zhang X, Tan J, Sun H and Ji W: MiR-138 regulates dendritic cells mediated Th2-type immune response by regulating the OX40L expression in asthma. Int J Clin Exp Pathol 10: 10979-10988, 2017.

35. Amrani Y, Tliba O, Choubey D, Huang CD, Krymskaya VP, Eszterhas A, Lazaar AL and Panettieri RA Jr: IFN-gamma inhibits human airway smooth muscle cell proliferation by modulating the E2F-1/Rb pathway. Am J Physiol Lung Cell Mol Physiol 284: L1063-L1071, 2003

36. Ren XS, Yin MH, Zhang X, Wang Z, Feng SP, Wang GX, Luo YJ, Liang PZ, Yang XQ, He JX and Zhang BL: Tumor-suppressive microRNA-449a induces growth arrest and senescence by targeting E2F3 in human lung cancer cells. Cancer Lett 344: 195-203, 2014.

37. Qiu YY, Wu Y, Lin MJ, Bian T, Xiao YL and Qin C: LncRNA-MEG3 functions as a competing endogenous RNA to regulate Treg/Th17 balance in patients with asthma by targeting microRNA-17/ ROR $\gamma$ t. Biomed Pharmacother 111: 386-394, 2019.

38. Liang $Z$ and Tang $F$ : The potency of lncRNA MALAT1/miR-155/CTLA4 axis in altering Th1/Th2 balance of asthma. Biosci Rep 40: BSR20190397, 2020. 